\title{
Stroke unities: the role of the occupational therapist and the perception of the multidisciplinary team
}

\begin{abstract}
Every second a person in the world suffers from a stroke, not surprising, therefore, that stroke is the leading cause of death and morbidity in Portugal. Increasingly, acute stroke is considered a medical emergency. The evidence proves that the treatment of these patients in specialized units (stroke units) is effective in acute stroke. A stroke unit is a hospital area where professionals with specific, well-defined training work, who provide care to stroke patients who are already stabilized, but are still in an acute phase(DGS, 2001). The aim of this study is to understand the role of the occupational therapist in stroke units and to identify the perspective of the multidisciplinary team on their work, clarifying what are the advantages of this professional in the team. The study falls within the qualitative paradigm, exploratory and descriptive. Semi-structured interviews were performed to 39 health professionals. The technique used was the content analysis of interviews. Based on previously established categories, other categories emerged.
\end{abstract}

Keywords: occupational therapy, stroke unities, health professionals, rehabilitation
Volume 6 Issue 4 - 202I

\author{
António Arsénio Duarte, ' Ana Paula \\ Martins,' Diana Santos, ${ }^{2}$ Rafael Santos, ${ }^{2}$ Rita \\ Viegas $^{2}$ \\ 'Teachers of the Degree in Occupational Therapy of the Beja \\ Polytechnic Institute, Portuga \\ ${ }^{2}$ Graduating Students of the Degree in Occupational Therapy of \\ Beja Polytechnic Institute, Portuga
}

Correspondence: António Arsénio Duarte, Beja Polytechnic Institute, Rua Pedro Soares, Apartado 6I55, Beja, Portugal, Email ajaduarte@gmail.com

Received: July 29, 202I | Published: November |8,202

\section{Introduction}

Stroke is a global health-care problem that is common, serious, and disabling. ${ }^{1}$ In Portugal, stroke is the first most common cause of death and one of the main causes of acquired adult disability. ${ }^{1}$ Its global prevalence in the general population ranges from $0.5 \%$ to $0.7 \% .^{2}$ Approximately $50 \%$ of the stroke population survives and requires health supervision after hospital discharge, often in primary healthcare, which demands orientation and care to adapt to the new lifestyle that the disease imposes. However, approximately $20 \%$ of stroke victims die within one month after the accident. An additional $30 \%$ survive and, despite certain neurological deficits, they remain able to live independently. ${ }^{3}$

A stroke occurs when the blood supply to part of your brain is interrupted or reduced, preventing brain tissue from getting oxygen and nutrients. Brain cells begin to die in minutes so, an early and specialized intervention is essential to minimize the sequelae that result from this pathology. New approaches to the problem were considered, which include better care during hospitalization, better follow-up at the clinic and coordination with other institutions, public or private, that provide their contribution to the patient and their families. ${ }^{1}$

Overall, in Portugal, there was an improvement in all indicators of cerebrovascular diseases because of the combined action of adopting preventive measures and the organization of health services. Emphasis is placed on the proper functioning of the Percutaneous Coronary Intervention Units in the Acute Myocardial Infarction, in close articulation with the emergency pre-hospital assistance system (Coronary Pathways and Stroke). Organised inpatient (stroke units) has been identified as one of the ways that have contributed most to these gains.

In 2012, 50\% of users hospitalized for stroke had access to specialized units. This percentage has doubled in the last 3 years, reflecting the large investment made in material and human resources, which allowed to widen the scope of the territorial coverage of Via Verde Coronária and the increase in users correctly referred by the pre-hospital emergency system, which has shown the narrow window of opportunity for the administration of thrombolysis treatment, applied until the first 3 hours in the case of Ischemic Stroke.

A recent study ${ }^{4}$ found moderate-quality evidence that stroke patients who receive stroke unit care are more likely to be alive, independent, and living at home one year after the stroke. The apparent benefits were independent of patient age, sex, initial stroke severity, or stroke type, and were most obvious in units based in a discrete stroke ward.

Stroke units are specialized centers in an acute hospital with dedicated beds, offering a multidisciplinary treatment of strokes by dedicated stroke teams addressing the acute treatment, early mobilization and rehabilitation as well as secondary prevention. This requires highly qualified staff, defined infrastructural equipment and well-organized procedures. Thus, organized stroke unit care is provided by multidisciplinary teams that exclusively manage stroke patients in a dedicated ward (stroke ward), with a mobile team (stroke team) or within a generic disability service (a mixed rehabilitation ward). The goals of stroke units are: (1) To continuously monitor parameters such as ECG, heart rate, blood pressure, respiration, oxygen saturation, temperature and glucose, in order to prevent and treat complications, (2) To provide thrombolysis treatment for patients within the time window of 3-4.5 hours, (3) To provide thrombectomy, if possible, (4) To identify stroke causes in order to provide a targeted treatment, (5) To provide early mobilization and rehabilitation and (6) To provide secondary prevention in order to prevent infarct progression and recurrent events

The stroke unit is currently the best place to treat the acute stroke patient. There is strong evidence that multiprofessional organized care reduces mortality, the need for institutionalization, permanent disability, as well as increasing recovery and independence in activities of daily living. ${ }^{1,4}$ To provide appropriate early mobilization and rehabilitation stroke units also need physiotherapists, speech therapists, occupational therapists and social workers. ${ }^{4}$ In cooperation with the stroke nurses, they are responsible for early mobilization, recognition and treatment of dysphagia, the organization of discharge management and the planning of post-acute rehabilitation. 
Rehabilitation is a user-centered and goal-oriented process It starts as soon as the clinical condition is stable $e^{1,5,6}$ and aims to improve functionality and achieve the highest level of independence possible. It goals go through at restoring possible impairments and treating comorbidities that have been responsible for stroke in order to prevent recurrent strokes. This also includes a well-organized discharge management and coordination with rehabilitation centers and outpatient physicians.

Multidisciplinary team working is regarded as fundamental to delivering effective care across the stroke pathway, especially during the rehabilitation process. ${ }^{4,8}$ Multidisciplinary rehabilitation is therefore a central tenet of high-quality stroke care. ${ }^{9}$ In these teams their members contribute different professional perspectives, but goal setting, care planning, and decision-making are collaborative activities. This understanding and acceptance of blurring of role boundaries facilitates rapid information exchange, enables early interventions, and underpins effective rehabilitation. Interdisciplinary teamwork implies that not only do team members perform activities toward a common goal, but they also accept the added responsibility of group effort on behalf of patients. ${ }^{10,11}$

For the team to function at its fullest, there needs to be effective communication between the different professionals and to know their role as a member of the team. The interdisciplinary communication is now a requirement and a challenge for all professionals, institutions and bodies that are engaged in cross-sectoral cooperation in health, because it allows more complete and effective care centered on patients' needs. However, this is the great challenge for multidisciplinary teams. Knowing how to communicate, as well as recognizing the limits of your performance and that of other team members, are key points for commitment to the team. ${ }^{8,11}$

Occupational therapy during rehabilitation focuses on ensuring that the client will function as well as possible after discharge, which often includes caregiver education and training, if needed, during post-rehab intervention. Other occupational therapy interventions include home modifications, assistive technology training, and wheelchair prescriptions (manual or powered) for improving quality of life and increasing independence. ${ }^{12}$ However, after stroke, occupational therapists work to facilitate and improve motor control and hand function in the stroke-affected upper limb; to maximize the person's ability to undertake his or her own personal self-care tasks and domestic tasks; to help the patient learn strategies to manage the cognitive, perceptual, and behavioral changes associated with stroke; and to prepare the home and work environment for the patient's return. ${ }^{13}$ For this purpose, the occupational therapy intervention is initially based on Assessment is conducted to understand the impact of changes in motor function, sensation, coordination, visual perception, and cognition on the stroke victim's capacity to manage daily life tasks. ${ }^{11}$ Considering that the occupational therapist is one of the elements of the multidisciplinary team that intervenes early with the stroke patient, it is important to know how the other professionals in the team perceive their role within the team.

\section{Material and methods}

A qualitative study design, using semi-structured interviews, was employed to obtain a rich and in-depth understanding of how health professionals perceive the role of the occupational therapist in stroke units, and how important they are while part of team. The importance of qualitative data in exploratory studies, where little is known about the subject, is recommended. ${ }^{14}$
The study was conducted in a convenient sample of three referral professional groups(Doctors, Nurses and Physiotherapists)in stroke units of two referral hospitals, one of them has occupational therapists (Centro Hospitalar do Algarve- CHUA), the other doesn't (Hospital José Joaquim Fernandes da Unidade Local de Saúde do BaixoAlentejo- ULSBA). Data from each group of interviews were analysed separately, recorded, and later transcribed without any kind of alteration, giving rise to the material that served as the basis for the content analysis.

All interviews took place individually, and is duration ranged from 30 to 45 minutes. The interviews were guided by a semi-structured interview framework, giving respondents a high degree of control over the conversation. Although each interview covered the same broad topics, new topics introduced by the interviewee were discussed in detail as they arose.

The interview script was initially tested in a pilot study involving six participants from each stroke unit. The interview itself was iterative and evolved (only by gaining items) over the course of the study.

No potential informant contacted refused to participate in the study. All participants were assured that their voluntary participation would remain anonymous.

\section{Results}

Interviews were carried out with 39 health professionals, with homogeneous distribution among doctors, nurses, and physiotherapists. 21 were from CHUA and 18 from ULSBA.

Both stroke services studied appeared to be heavily underpinned by a neurological service model. This had clearly produced significant benefits in driving the development of acute stroke care through prompt access to hospital and developing diagnostic imaging techniques and thrombolysis (especially the stroke unit of the CHUA). It appeared that the specialist service model adopted on the stroke units had benefits for the development of multidisciplinary staff knowledge and expertise ("....your knowledge advances so much quicker because yousee (strokes) more often ..." says a doctor).

Professionals from both units refer to the importance of a multidisciplinary team, reinforced by doctors, nurses and physiotherapists, where each has its role but, globally, reinforces the role of others. The importance of a team approach to the delivery of the stroke services, include the Occupational Therapy, was recognized by nursing staff, which appeared to have improved with the co-location of stroke patients in a specific clinical area. The professionals at the stroke unit ULSBA interviewed, mention that there are organizational failures that end up "forcing" the nurse to replace the functions of Speech Therapy, Physiotherapy and Occupational Therapy.

A range of issues was identified as representative of the occupational therapy contribution to multidisciplinary care. These included providing a family perspective, advocacy for the patient, assessment, and discrete clinical or functional elements such as mobility, cognition and sensorimotor components that compromise performance in activities of daily living.

The nurses of CHUA refer three clinical issues in which nurses and therapists collaborated provided good examples of differences multidisciplinary working: dressing practice, feeding, and patient moving and handling. In this unit, doctors also consider that the occupational therapist's intervention is global and that it goes beyond 
assessment, rehabilitation, and psychological monitoring. It plays an important role in preparing for discharge and accompanying the family.

The ULSBA nurses, on the other hand, although they do not have occupational therapists in the team, believe that these professionals could bring a different dynamic to the multidisciplinary team, freeing them to do their tasks better. According to them, the organizational failures, and the lack of elements in the team, end up "forcing" the nurse to replace the Occupational Therapy functions. One nurse said it was hard to do rehabilitation on the unit because of limited time and space: "There are limitations on what we can do in the time and space allotted. To be able to come in and have the time to do all of those extra things...like assist patients in their room with feeding or mobilization and all of those things...that would be great, but it doesn't happen".

Doctors think that the occupational therapist who works in these services has an integral role that includes assessment, identification and monitoring, in addition to rehabilitation and psychological monitoring, saying that it would be an asset to have them as a member of the multidisciplinary team.

The physiotherapists at CHUA refer that the occupational therapist "is an asset in the acute phase of the user because it complements the work of all professionals". Its intervention ranges from cognitive rehabilitation to sensory motor, playing a very important role in the study of support products and orthotics. One of the physiotherapists reported "...Occupational therapists are experts in measuring functional cognition, which encompasses assessment of everyday task performance... are experts in identifying performance-based cognitive impairments, which range from subtle to obvious".

All participants stated that communication and collaboration between all members of the team is essential both for the success of the unit and for the patient's survival. The stroke unit was supposed to be an interdisciplinary place where staff felt comfortable sharing information. It would work in a non-hierarchical way, with each professional's opinion being valued and forming part of the plan for medical and rehabilitative care. However, some nurses felt that therapists' failure to recognize and acknowledge the role of nurses in rehabilitation can lead toresentment and halfhearted attempts at rehabilitation. They felt that therapists had a narrow view of nursing practice (during their professional interactions, nurses were responsible for patients' medical needs while therapists were responsible for rehabilitation).One nurse lamented: "We are not recognized for the mobility things we do or in any concerns we have about our patients...".

To demystify this idea, communication between all members of the team was very important, clarifying the role of each profession and how they could be helped, as a working group, in favor of better patient care. All members of the CHUA stroke unit team currently congratulate themselves on the relationship that exists between the team, the respect for their professions and, above all, the respect they have for the patient and his family and for the fact that they place them at the center of its activities.

\section{Discussion}

As referred to in the literature and studies in the area, most participants in this study consider that if patients are treated in stroke units, there is strong evidence that the levels of mortality, morbidity and dependence can decrease considerably. The likely reasons for this to happen are mainly due to three aspects: intensive approach to managing medical complications, early and more patient-centered rehabilitation and a multidisciplinary approach. . $, 4,5,8,10,11^{-1}$

There is unequivocal evidence of improved outcomes when patients are treated in a stroke unit by multi -disciplinary teams. There is also good evidence that teams facilitate earlier discharge to the home, increase the likelihood that patients will regain independence in activities that support daily living, and result in fewer patients requiring long-term institutional care. ${ }^{5,8,9}$

For the team to function at its fullest, it is necessary to have effective communication between the different professionals and to know their role as a member of the team. When this does not happen, the literature highlights many problems with teamwork, including petty jealousy, ignorance, and a perceived loss of autonomy and threat to professional status. ${ }^{811}$ Fortunately, this is not the case for the teams from both units studied. All participants stated that communication and collaboration among all team members is essential and effective in their work units. However, some statements can be considered ambivalent, namely when the professionals at the stroke unit ULSBA interviewed, mention that there are organizational failures that end up "forcing" the nurse to replace the functions of Speech Therapy, Physiotherapy and Occupational Therapy, not realizing if these refer to an increase in their work or if the professionals mentioned are essential to the team. However, all the team members of the CHUA stroke unit are congratulated for the relationship that exists between the team and, above all, for the respect that exists for their professions. The lack of human resources also comes as an obstacle to the proper functioning of the units, with the absence of sufficient Nurses and the lack of elements in the rehabilitation team, including Occupational Therapists, highlighted.

A range of issues was identified as representative of the Occupational Therapy contribution to multidisciplinary care. These included providing a family perspective, advocacy for the patient, assessment, and discrete clinical or functional elements such as mobility, cognition and sensorimotor components that compromise performance in activities of daily living.

It is essential that each professional recognizes both their role and that of others, as members of a multidisciplinary team. The skills of each one adds diversity to the team and enhance their intervention, namely through different assessments and approaches. ${ }^{12,13}$ Regarding the role of the Occupational Therapist it was the Physiotherapists who came closest to their functional contentes ${ }^{11,12,13}$ but with a vision, although focused on functionality, not very comprehensive because only aspects related to physical rehabilitation were mentioned. The CHUA team was the one that best identified the Occupational Therapist's population, reinforcing its contribution to the multidisciplinary team. This included providing a family perspective, patient advocacy, assessment, and distinct clinical or functional elements such as mobility, cognition, and sensorimotor components that compromise performance in activities of daily living.

The CHUA teams develop, in a coordinated manner, procedures to formulate and apply a care plan, including rehabilitation, based on the patient's needs and problems, on the notion of stroke as a medical emergency and on the great collaboration between the various specialties. $^{2}$

\section{Conclusion}

Occupational Therapy professionals collaborate closely with other health care professionals such as physicians, nurses, physiotherapists, and speech therapists to create an appropriate, coordinated, 
interdisciplinary plan of care and discharge plan.They have a good understanding of the match between the patient's needs, abilities, and the environments in whichtheyneed to function, which assists with the successful transition to the home, community, or next level of care.

Occupational therapy interventions are in accordance with the needs, interests, and values that are of importance to the clients. To this end, occupational therapists offer a unique and holistic approach to enhance or enable participation in daily life activities. They use therapeutic activities by identifying client problems, goals, and treatment focus to improve independence in life skills and to promote quality of life but Occupational Therapy also plays a key role in the lives of people dealing with disabling or potentially disabling conditions, specifically caregivers.

Most participants in this study identified, albeit partially, the role of the Occupational Therapist in the multidisciplinary team of stroke units, however, there are still some individuals in both institutions who are profoundly unaware of what Occupational Therapyis, what is its role in hospitalization and what was the difference between the professional areas of Occupational Therapy and Physiotherapy. Although these are in short supply, it is still worrying that health professionals do not know their peers, both what brings them together and what differentiates them and how a complete and diverse team enhances the effectiveness of the treatment and the satisfaction of patients and of their families.

It is up to Occupational Therapists to effectively publicize their profession and, above all, through their work to prove that stroke units are also more effective if they have these professionals in their teams.

\section{Acknowledgments}

We thank the staff and management in the stroke services who gave their time to contribute to the study.

\section{Conflicts of interest}

The authors report no conflicts of interest in this work.

\section{References}

1. Direção-Geral de Saúde. Portugal Doenças Cérebro-Cardiovasculares em Números. Lisboa: Direção-Geral de Saúde.2015.
2. Warlow CP, Van Gijn J, Dennis MS, et al. Stroke: practical management $3^{\text {rd }}$ ed. Oxford: Blackwell Publishing; 2008.

3. Rodgers H. Risk factors for first-ever stroke in older people in the North East of England: a population based study. Stroke. 2004;35(1):7-11.

4. Carolee J, Winstein, Joel Stein, et al. Guidelines for adult stroke Rehabilitation and recovery A guideline for healthcare professionals from de American Heart Association/American Stroke Association. Stroke. 2016;47.

5. Langhorne P, Ramachandra S. Organised inpatient (stroke unit) care for stroke: network meta-analysis. Cochrane Database of Systematic Reviews. 2020;4.

6. Cancela DM. O Acidente Vascular Cerebral_Classificação, Principais Consequências e Reabilitação. Porto: Universidade Lusíada: ESO.2008.

7. European Stroke Organization. Recomendações para o Tratamento do AVC Isquémico e do Acidente Isquémico Transitório. Alemanha: ESO. 2008.

8. Clarke DJ, Forster A. Improving post-stroke recovery: the role of the multidisciplinary health care team. Journal of multidisciplinary healthcare. 2015;8:433-442.

9. Clarke DJ. The role of multidisciplinary team care in stroke rehabilitation. Prog Neurol Psychiatry. 2013;17(4):5-1

10. McCallin AM, McCallin MCH. Factors affecting successful team working and strategies to facilitate successful collaborative teamwork. $N Z$ J Physiother. 2009;37:62-68.

11. Clarke DJ. Achieving teamwork in stroke units: the contribution of opportunistic dialogue. J Interprof Care. 2010;24(3):285-97.

12. American Occupational Therapy Association. Occupational therapy practice framework: Domain and process (3rd ed.). American Journal of Occupational Therapy. 2014;68:S1-S48.

13. Rowland TJ, Cooke DM, Gustafsson LA. Role of occupational therapy after stroke. Ann Indian Acad Neurol. 2008;11(S1):99-107.

14. Greenhalgh T, Howick J, Maskrey N. Evidence based medicine: a movement in crisis? BMJ. 2014;348-372. 